\title{
Las familias en el corazón de la educación inclusiva ${ }^{1}$
}

\section{Cecilia Simón Rueda y Ángela Barrios Fernández}

Universidad Autónoma de Madrid - España

\section{RESUMEN}

El trabajo que se presenta analiza cómo se entiende el papel que las familias deben desempeñar en la transformación de los sistemas y entornos educativos para ser más inclusivos. Para ello, partiendo del significado de una educación inclusiva, y considerando esta como un derecho de todo el alumnado sin exclusiones, se justifica por qué las familias deben formar parte de este proceso, así como los beneficios que se derivan de su participación real en los centros escolares. Asimismo, a partir de experiencias de centros escolares que comparten los valores de la educación inclusiva, se muestra qué lugar han ocupado las familias en ellos. Finalmente, se realiza una reflexión en torno a qué tipo de participación por parte de las familias es necesario promover para acortar la distancia entre los valores declarados respecto a la inclusión y las acciones que se llevan a cabo, así como las oportunidades y espacios que se ofrecen a las mismas en los centros escolares.

Palabras Clave: Educación inclusiva, derecho, participación, voces de las familias.

\section{Families at the heart of inclusive education}

\section{ABSTRACT}

The paper analyses the role that families must play in transforming education systems and settings to be more inclusive. Therefore, based on the meaning of inclusive education, and considering this to be a right for all students without exclusions, the reason why families should be part of this process can be justified, as well as the benefits derived from their real participation in schools. Similarly, based on the experiences of centres that share the values of inclusive education, the place families have occupied is shown. Finally, we will reflect about what type of participation by families is necessary to promote in order to reduce the gap between the stated values with respect to inclusion and the action that are carried out, as well as the opportunities and spaces that are offered to families in schools.

Keywords: Inclusive education, rights, participation, family voices.

\section{Introducción: breve acercamiento al derecho a una educación inclusiva}

En este momento nadie pone en duda que la educación inclusiva es un derecho de todos los alumnos y alumnas (IBE-UNES$\mathrm{CO}, 2016)$. Aunque no dispongamos de una definición consensuada, sí contamos con acuerdos en torno a los elementos centrales que la configuran. Atendiendo al IBE-UNESCO (2016) entendemos la educación inclusiva como: a) un proceso, un compromiso de toda la comunidad educativa para buscar continuamente cómo responder de forma más adecuada a este derecho. Ello implica ver la diversidad como una oportunidad para mejorar las prácticas y los entornos educativos (UNESCO, 2005); b) la articulación de tres dimensiones como son la presencia, el aprendizaje y la participación (entendida también por la atención al bienestar emocional y social del alumnado); c) la preocupación por todos los estudiantes sin excepciones, a la vez que se está muy atento a aquellos que, por diferentes circunstancias, pueden encontrarse en una situación de especial riesgo de exclusión; d) la identificación de las barreras que limitan la presencia, el aprendizaje y la participación, que se encuentran en la culturas, las políticas y las prácticas escolares; así como la puesta en marcha de procesos de innovación y mejora escolar, encaminados a eliminarlas.

Como señalan Porter y Towel (2017), hablamos de la creación de entornos de aprendizaje que maximizan el potencial de cada persona en nuestras sociedades diversas, con la finalidad de recibir una educación de alta calidad en las escuelas que se encuentran en la comunidad local. Esta tarea se ha convertido en la ruta reconocida internacionalmente que deben trazar los siste-

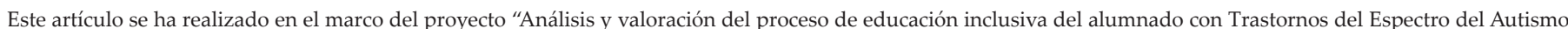

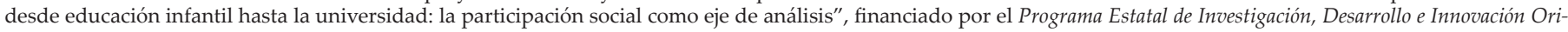
entada a los Retos de la Sociedad. Ministerio de Economía, Industria y Competitividad (EDU 2017-86739-R)
} 
mas educativos para mejorar la equidad en la educación. Como se recoge en el objetivo número 4 de la Agenda 2030 para el Desarrollo Sostenible ${ }^{2}$, nos encontramos ante el reto internacional de "Garantizar una educación inclusiva, equitativa y de calidad, y promover oportunidades de aprendizaje a lo largo de toda la vida para todos y todas". Conforme señala la UNESCO (2016), la educación está en el centro de la agenda de desarrollo sostenible y es esencial para el éxito de todas las metas. La nueva agenda de educación resumida en este Objetivo 4 está inspirada por una visión de que la educación transforma las vidas de las personas, las comunidades y las sociedades.

Por ello, la pregunta que debemos plantearnos es ¿cómo podemos hacer realidad este derecho? Debemos asumir que este proceso requiere de una transformación sistémica que afecta a la educación en todos sus niveles. Como señala la UNESCO (2005, p. 13), la educación inclusiva "representa una perspectiva que debe servir para analizar cómo transformar los sistemas educativos y otros entornos de aprendizaje, con el fin de responder a la diversidad de los estudiantes". Aunque en este texto centraremos la atención en torno al ámbito escolar, consideramos necesario señalar que, de acuerdo con la reflexión realizada por Ainscow, Dyson, Hopwood y Thomson (2016) respecto a lo que denominan "ecología de la equidad", la educación inclusiva no es responsabilidad únicamente de "la escuela", sino de la sociedad en su conjunto. Es preciso considerar factores de la propia escuela, los vínculos existentes entre escuelas, pero también otros más allá de las mismas y que tienen que ver, por ejemplo, con las políticas de protección a la infancia o el contexto en el que estas se encuentran. Aspectos todos ellos que se influyen mutuamente.

En último término, el cambio de las prácticas de exclusión a las de inclusión nos habla de construir una sociedad más justa y nos plantea en qué clase de mundo queremos vivir juntos (Booth y Ainscow, 2015). De hecho, contamos con evidencias que muestran que la educación inclusiva beneficia a todas las personas que participan en los entornos educativos. Así, por ejemplo, atendiendo únicamente al desempeño del alumnado en los centros escolares, la revisión de la literatura internacional realizada por Hehir et al. (2016) muestra los efectos positivos de la educación en contextos inclusivos, tanto desde un punto de vista académico como en el desarrollo socioemocional, en este caso, para estudiantes con y sin discapacidad. De acuerdo con autores como Ainscow, Dyson, Goldrick y Kerr (2015) o Echeita (2017), la necesidad de promover el aprendizaje y la participación de la diversidad de estudiantes puede actuar como catalizador de la puesta en marcha de procesos de innovación y mejora escolar que, sin duda, redundarán en la mejora del centro en general y de la actuación docente en particular (por ejemplo, en las metodologías de enseñanza).

El foco de atención en este momento es: ¿cuáles son las claves que están facilitando la transformación de los sistemas educativos y los centros con la finalidad de ser más inclusivos? En este texto nos centraremos en una de ellas, como es la de promover la colaboración entre los diferentes agentes implicados (Booth y Ainscow, 2015; Porter y Towell, 2017; Puigdellívol et al., 2017) para tratar específicamente el rol que desempeñan las familias en este ineludible proceso.

\section{La familia como agente necesario en los procesos de transfor- mación de las escuelas con la finalidad de ser "más inclusivas"}

Las familias están "en el corazón de la educación inclusiva", como destacan Porter y Smith (2011), de ahí el título de este ar- tículo. Las familias y las organizaciones civiles pueden jugar un papel de gran relevancia en el impulso de políticas educativas alineadas con la inclusión, como de hecho así ha ocurrido en diferentes países (UNICEF, 2014).

Asimismo, si atendemos a las claves que se han destacado en la transformación de los sistemas educativos para hacer efectivo el derecho a una educación inclusiva, las familias están, de una u otra forma, presentes en las mismas. Por ejemplo, entre las diez que proponen Porter y Towell (2017) para este fin, se encuentra “establecer alianzas. Más allá del liderazgo profesional, necesitamos reconocer que las escuelas son una parte vital de la comunidad y que mejorar la educación implica trabajar en alianza con los estudiantes y sus familias" (p. 9). La implicación de las familias y la comunidad en los centros escolares es un principio de calidad educativa (UNICEF, 2014). En esta línea, como señalan Leiva y Escarbajal (2011), es necesario reconocer el importante papel que deben tener las familias en la construcción de una sociedad democrática intercultural. Reto necesario que los centros deben asumir, sabiendo aprovechar la riqueza de la diversidad de familias, con diferentes situaciones, procedencias, bagajes culturales, etc., si realmente queremos construir una escuela para todos y todas.

De acuerdo con Porter y Smith (2011), respetar y honrar la voz y las perspectivas de las familias es un componente esencial de la educación inclusiva. Las instituciones que comparten creencias respecto a que las familias son un gran valor, fuente de recursos, parte de la comunidad, miembros igualitarios y corresponsables en su labor educativa, se aseguran de invitarlas e incluirlas en los procesos de enseñanza y aprendizaje, así como en los diferentes espacios de la escuela. Todo ello, como proponen Collet-Sabé, Xavier, Feu y Tort (2014), debe hacerse desde una propuesta de actuación basada en la inclusión de todas las familias, poniendo el foco en el establecimiento de un buen vínculo con ellas, y desde un compromiso con la lucha contra la reproducción de las desigualdades sociales y por el éxito escolar de todo el alumnado. Esto implica la responsabilidad del docente en esa tarea, así como un planteamiento de corresponsabilidad educativa. La auténtica inclusión de las familias es un componente irrenunciable en las prácticas de este profesorado. De la misma forma, los docentes que no reconocen o respetan las experiencias y conocimientos de estas, que no las consideran un recurso educativo valioso, pueden implícita o explícitamente excluirlas, con la consiguiente pérdida de recursos que ello conlleva. Conocemos los beneficios de la implicación de las familias en las escuelas para todos los miembros de la comunidad educativa (Epstein, 2011). En este sentido, cabe destacar la gran relevancia que cobra y los beneficios que de ella se desprenden en el caso del alumnado en situación de especial vulnerabilidad (Ainscow et al., 2016; Rodrigo, Martínez-González y Rodríguez-Ruiz, 2018).

Como recogen Simón, Giné y Echeita (2016a), las familias son un gran recurso para apoyar al profesorado en su actuación docente y a la escuela en los procesos de mejora. Los padres, madres o tutores, así como otros miembros de la familia, disponen de información, talentos y perspectivas que son de gran utilidad para que el centro educativo alcance el objetivo común de optimizar el aprendizaje y la participación de cualquier alumno o alumna. Tienen experiencias y conocimientos sobre sus hijos e hijas que complementan a los de los profesionales y que pueden ayudarles en el ajuste de sus prácticas. En lo que respecta a la mejora del centro, son de gran valor para la identificación de las barreras y los facilitadores que afectan a la presencia, al aprendizaje y a la participación de los alumnos y alumnas, así como para los pro-

https://es.unesco.org/sdgs 
cesos de planificación e implementación de las iniciativas encaminadas a la mejora e innovación escolar. También pueden ser un gran apoyo para las propias familias (Gallego, 2013; Gallego, ذiménez y Corujo, 2018; UNICEF, 2014), lo que puede redundar en la mejora de la calidad de vida de todas ellas (Brown, Cobigo y Taylor, 2015) y suponer un recurso para la propia comunidad local, convirtiendo la escuela en un motor de mejora del entorno (Parrilla, Muñoz y Sierra, 2013).

Un indicador muy claro de que la educación inclusiva no es posible si no se cuenta con las familias, sin su implicación real (Parsons et al., 2018) es el espacio reservado al análisis y promoción de su implicación en las guías encaminadas a ayudar y orientar a los centros escolares en el proceso de su transformación, con la finalidad de ser más inclusivos. Con la pretensión de ilustrarlo, lo analizaremos en cuatro guías diferentes: $\underline{\mathrm{Al}}$ berta Education (2013), Booth y Ainscow (2015), CSIE (2016) e IBE-UNESCO (2016).

En ellas se recogen dimensiones, indicadores o preguntas que permiten a los centros identificar tanto barreras como facilitadores para planificar los oportunos procesos de mejora. Hemos agrupado las cuestiones que incluyen algunas de estas guías en torno a dos aspectos que tendrían que ver tanto con la cultura, como con las políticas y prácticas de los centros: cómo se debe contemplar el papel de las familias y qué tipo de relación y participación se ha de promover.

En general, en cuanto al papel que se otorga a las familias, podríamos resumir que se consideran parte activa de la comunidad educativa y agente necesario si se pretende construir entornos acogedores para todas las personas, en el marco de culturas escolares alineadas con los valores y principios de la inclusión. En relación con este aspecto, se reconoce que el centro debe ser sensible a la diversidad de las familias, a sus necesidades, intereses, opiniones, así como a las circunstancias de cada una de ellas. Se pone en evidencia la responsabilidad de crear oportunidades para que las familias se sientan parte, se encuentren a gusto y participen de una forma real en el centro. Los documentos recogen condiciones necesarias para establecer una relación positiva con las familias como, por ejemplo, que sean bienvenidas y se sientan acogidas, que la información de la escuela sea accesible para ellas y que se genere un clima de confianza y compromiso, atendiendo también a su bienestar.

A modo de ejemplo, en la Tabla 1 se muestran algunos indicadores o preguntas incluidas en estas herramientas en relación con este aspecto, y que se han organizado en torno a lo que hemos denominado "facilitadores" ${ }^{3}$ :

Tabla 1.

Facilitadores identificados respecto al papel que se otorga a las familias en los centros

FACILITADOR

\begin{tabular}{cl}
\hline & $\begin{array}{l}\text { Sensibilidad del centro a la diversidad, necesidades y } \\
\text { circunstancias de las familias. } \\
\text { Asegurar que todos los aspectos de la diversidad, in- } \\
\text { cluyendo a toda la diversidad de familias, son valora- } \\
\text { dos, celebrados y utilizados como un valioso recurso } \\
\text { para el aprendizaje; prevenir la posibilidad de que al- } \\
\text { guien se sienta inseguro, denigrado o menospreciado. }\end{array}$ \\
$\begin{array}{c}\text { Valoración de } \\
\text { la diversidad y } \\
\text { preocupación por el } \\
\text { bienestar de las familias }\end{array}$ & $\begin{array}{l}\text { Recordar que algunas familias tienen diferentes expe- } \\
\text { riencias educativas y pueden tener diferentes expec- } \\
\text { tativas }\end{array}$ \\
& ¿Todos se sienten enriquecidos por la diversidad? \\
& \\
& $\begin{array}{l}\text { ¿Todos los agentes (incluidas las familias) disponen } \\
\text { de tiempo para hablar de valores y diversidad? }\end{array}$
\end{tabular}

¿Se procura que las familias se sientan bienvenidas al llegar a la escuela?

¿Las familias, junto con la dirección y los estudiantes, crean un sentido de comunidad en el centro?

Información sobre la comunidad escolar accesible para las familias.

Disponer de los documentos importantes traducidos a los principales idiomas de la comunidad local y contar con intérpretes en las reuniones de familiares...

\section{GUÍA: DIMENSIÓN APARTADO O SECCIÓN}

Alberta Education (2013): Dimensión 1. Establecer valores y principios inclusivos

CSIE (2016): Logro

CSIE (2016): Bienestar

Booth y Ainscow (2015):

A. Creando culturas inclusivas. El centro escolar anima a los estudiantes y a los adultos a sentirse bien consigo mismos

Booth y Ainscow (2015):

A. Creando culturas inclusivas. El centro escolar desarrolla valores inclusivos compartidos.

IBE-UNESCO (2016): Guía 2. Todos se sienten bienvenidos

Booth y Ainscow (2015):

A. Creando culturas inclusivas. Todo el mundo es bienvenido

Alberta Education (2013): Dimensión 5. Compromiso con las familias y la comunidad

CSIE (2016): Liderazgo

\begin{abstract}
Accesibilidad a escuela y su comunidad
\end{abstract}

sión de que son absolutamente necesarias y que todas cuentan con fortalezas para contribuir a la trasformación del centro para ser cada vez más inclusivo (ver Tabla 2). ha de promoverse, las guías nos muestran que las familias deben ser un miembro igualitario de la comunidad educativa, recurso y apoyo para el profesorado y el centro escolar, desde la compren- 


\section{FACILITADOR}

\section{INDICADOR O PREGUNTA}

\section{GUIA: DIMENSION APARTADO o SECCIÓN}

\begin{tabular}{|c|c|c|}
\hline \multirow{3}{*}{$\begin{array}{l}\text { La escuela reconoce las } \\
\text { fortalezas de las familias } \\
\text { y las capacita para su } \\
\text { participación }\end{array}$} & $\begin{array}{l}\text { La escuela valora y promueve el compromiso y la par- } \\
\text { ticipación de las familias. }\end{array}$ & $\begin{array}{l}\text { Alberta Education (2013): Dimensión 5. Compromiso con las } \\
\text { familias y la comunidad }\end{array}$ \\
\hline & $\begin{array}{l}\text { ¿Se reconoce a las familias como socios para apoyar la } \\
\text { educación de sus hijos e hijas? }\end{array}$ & $\begin{array}{l}\text { IBE-UNESCO (2016): Guía 1. Dimensión 3. Apoyo de calidad } \\
\text { para grupos vulnerables de estudiantes }\end{array}$ \\
\hline & $\begin{array}{l}\text { Promover y dar la bienvenida a la participación de } \\
\text { todos los miembros de la comunidad educativa en la } \\
\text { toma de decisiones sobre la mejor manera de promo- } \\
\text { ver la igualdad en la escuela. }\end{array}$ & CNSE (2016): Liderazgo \\
\hline \multirow{4}{*}{$\begin{array}{c}\text { Colaboración en } \\
\text { el aprendizaje y la } \\
\text { participación del } \\
\text { alumnado (dentro y fuera } \\
\text { del aula) }\end{array}$} & $\begin{array}{l}\text { Profesionales y familias colaboran para apoyar el éxi- } \\
\text { to de los estudiantes. }\end{array}$ & $\begin{array}{l}\text { Alberta Education (2013): Dimensión 5. Compromiso con las } \\
\text { familias y la comunidad }\end{array}$ \\
\hline & $\begin{array}{l}\text { Ayudar a las familias a sentirse bienvenidas en la es- } \\
\text { cuela como expertas en sus propios hijos e hijas y so- } \\
\text { cios iguales en el apoyo a su aprendizaje y desarrollo. }\end{array}$ & CSIE (2016): Liderazgo \\
\hline & $\begin{array}{l}\text { ¿Todas las políticas de apoyo se negocian con las } \\
\text { familias? }\end{array}$ & $\begin{array}{l}\text { Booth y Ainscow (2015): } \\
\text { B. Establecer politicas inclusivas: Todas las formas de apoyo es- } \\
\text { tán coordinadas }\end{array}$ \\
\hline & $\begin{array}{l}\text { ¿Saben las familias cómo pueden apoyar el } \\
\text { aprendizaje de sus hijos e hijas? }\end{array}$ & $\begin{array}{l}\text { Booth y Ainscow (2015): } \\
\text { A. Creando culturas inclusivas: El equipo educativo y las fami- } \\
\text { lias colaboran }\end{array}$ \\
\hline \multirow[b]{2}{*}{$\begin{array}{l}\text { Colaboración en los } \\
\text { procesos de mejora } \\
\text { e innovación escolar } \\
\text { (evaluación del proceso } \\
\text { de educación inclusiva, } \\
\text { detección de barreras, } \\
\text { planificación de mejoras, } \\
\text { etc.) }\end{array}$} & $\begin{array}{l}\text { Invitar a todos los miembros de la comunidad educa- } \\
\text { tiva a evaluar el entorno escolar desde sus perspecti- } \\
\text { vas y hacer sugerencias de mejora si lo desean. }\end{array}$ & CSIE (2016): Entorno de aprendizaje \\
\hline & $\begin{array}{l}\text { ¿Hay un plan de mejora para el centro escolar y su } \\
\text { entorno que es ampliamente conocido y acordado por } \\
\text { el equipo educativo, el consejo escolar, las familias } \\
\text { y los estudiantes? ¿Se recogen las opiniones de los } \\
\text { estudiantes, las familias y del consejo escolar sobre } \\
\text { la naturaleza de las barreras para el aprendizaje y la } \\
\text { participación y sobre cómo puede mejorar el centro } \\
\text { escolar? }\end{array}$ & $\begin{array}{l}\text { Booth y Ainscow (2015): B Estableciendo politicas inclusivas: } \\
\text { El centro escolar tiene un proceso de desarrollo participativo }\end{array}$ \\
\hline \multirow{2}{*}{$\begin{array}{l}\text { Redes de apoyo para y } \\
\text { entre familias }\end{array}$} & $\begin{array}{l}\text { ¿Hay lugares donde las familias se reúnen para } \\
\text { intercambiar ideas? }\end{array}$ & $\begin{array}{l}\text { Booth y Ainscow (2015): A Creando culturas inclusivas: El } \\
\text { equipo educativo y las familias colaboran }\end{array}$ \\
\hline & $\begin{array}{l}\text { ¿El centro organiza clases para las familias y } \\
\text { miembros de la localidad? }\end{array}$ & $\begin{array}{l}\text { Booth y Ainscow (2015): A Creando culturas inclusivas: El } \\
\text { centro escolar y la comunidad se apoyan entre sí }\end{array}$ \\
\hline \multirow{2}{*}{$\begin{array}{l}\text { Naturaleza de la relación } \\
\text { de colaboración: } \\
\text { comunicación, confianza, } \\
\text { compromiso, igualdad, etc. }\end{array}$} & $\begin{array}{l}\text { Los profesionales, familias y agentes externos cola- } \\
\text { boran y se comunican con respeto y a través de vías } \\
\text { significativas. }\end{array}$ & $\begin{array}{l}\text { Alberta Education (2013): Dimensión 2. Construir entornos de } \\
\text { aprendizajes inclusivos }\end{array}$ \\
\hline & $\begin{array}{l}\text { Los profesionales, estudiantes y familias comparten } \\
\text { el compromiso de crear experiencias de aprendizaje } \\
\text { inclusivas para todos los estudiantes. }\end{array}$ & $\begin{array}{l}\text { Alberta Education (2013): Dimensión 1: Establecer valores y } \\
\text { principios inclusivos }\end{array}$ \\
\hline
\end{tabular}

En este caso se les otorga un papel activo en los procesos de enseñanza y aprendizaje dentro del aula, en otros contextos del centro y del hogar, así como en los procesos de innovación y mejora escolar, pero desde un modelo democrático de relaciones entre docentes y familia como señala Collet-Sabé et al. (2014). Precisamente, esta comprensión de la familia como apoyo se vincula a la forma más amplia y sistémica de entender los apoyos por parte de Booth y Ainscow (2015), como toda actuación que se lleva a cabo para responder con equidad a la diversidad del alumnado. Se entiende que las familias, lejos de adoptar un papel pasivo a modo de receptores de servicios, deben tener una responsabilidad compartida en estas acciones, por lo que el centro tendría que generar oportunidades para promover este tipo de implicación. En este sentido, cuando se reflexiona sobre el tipo de liderazgo que necesitamos en estos centros escolares, se habla de un liderazgo distribuido, que no se centra en una única figura, sino que se considera una tarea compartida en la que se busca la participación de los diferentes miembros de la comunidad educativa (Harris, Carrington y Ainscow, 2017).

Como hemos mostrado, la participación de la familia es un componente esencial de los instrumentos o guías para la revisión y mejora de las culturas, políticas y prácticas de los centros escolares para ser más inclusivos. A continuación, trataremos de analizar cómo se contempla, de hecho, esta participación en diferentes experiencias de centros escolares.

\section{Experiencia de centros escolares: qué papel se ha dado a las familias}

En este apartado pretendemos dar un paso más y mostrar cómo diferentes centros entienden el papel de las familias en la escuela, el sentido de su participación y en qué ámbitos se concreta. Para ello, se ha realizado una revisión y posterior selección de artículos con los siguientes criterios: estudios o experiencias 
de centros escolares de educación infantil, primaria y secundaria, que comparten los valores que fundamentan la educación inclusiva (Booth y Ainscow, 2015); trabajos de ámbito nacional e internacional, y análisis que muestren la actuación de centros en los que se encuentra alumnado en situación de especial riesgo de exclusión educativa o social. Si bien no pretendemos hacer una revisión exhaustiva, sí queremos acercarnos a la realidad de centros que se enriquecen de la diversidad de las familias que los conforman, presentando una atención especial a aquellas cuyos hijos e hijas, por diferentes circunstancias (sociales, económicas, personales, etc.), se encuentran en una situación de especial vulnerabilidad.

Las experiencias revisadas nos muestran que estos centros, en coherencia con los análisis que se han realizado en el apartado anterior:

a) Se preocupan por conocer a sus familias y el entorno de la escuela.

Se trata de centros que promueven la reflexión dentro de los mismos, con la finalidad de ayudar al profesorado, familias y alumnado a identificar dónde se sitúan con respecto a sus valores, principios y actitudes hacia la diversidad, así como conocer qué prácticas y estrategias contribuyen o no a mejorar la atención a la misma (Gallego, 2013). Por ello, consideran de gran valor reforzar el sentido de pertenencia al centro por parte de todos los miembros de la comunidad educativa, y son conscientes de las dificultades de las familias, de sus necesidades, demandas y prioridades (Egido y Bertran, 2017; Flecha, García y Rudd, 2011; Martínez-Figueira, Páramo-Iglesias y de Matos, 2015; Vigo, Dieste y Thurtson, 2016). También se interesan por las expectativas de estas respecto a sus propios hijos e hijas (en la medida que se entienden como barreras o facilitadores) para actuar sobre ellas (Labajos, Gallego y Lago, 2012; Martínez, Conroy y Cerreto, 2012). Todo ello se vincula con la puesta en marcha de acciones que les ayudan a delimitar procesos de mejora e innovación escolar en esta dirección (Martínez-González, Rodríguez-Ruiz y Gimeno, 2010).

b) Reconocen, valoran y aprecian a las familias como un activo para el centro escolar.

Tanto el equipo directivo como el profesorado comparten actitudes positivas respecto a su implicación, entendiendo que son necesarias para una práctica docente más ajustada a las necesidades del alumnado (De Verdier y Ek, 2014; Fernández-Batanero, 2014; Lyons, Thompson y Timmons, 2016). Se cuenta con equipos directivos que reconocen y valoran lo que las familias pueden aportar, que lideran procesos de construcción de una cultura escolar en donde este es un aspecto definitorio de la misma (Francis et al., 2016).

Como consecuencia de lo anterior, estos centros se preocupan por conocer los recursos y fortalezas de todas ellas (Martínez-Figueira et al., 2015). Saben aprovechar los "fondos de conocimiento e identidad" de familias y alumnado para mejorar los procesos de enseñanza y aprendizaje fomentando, asimismo, los vínculos entre escuela, familia y comunidad (Esteban-Guitart, Oller y Vila, 2012). Para ello, se presta atención a las familias desde los primeros momentos de su llegada al centro. Se cuidan los procesos de acogida a las familias, facilitando que se sientan bienvenidas (por ejemplo, con jornadas de puertas abiertas, jornada de acogida antes y después de la escolarización en el centro, entrevistas previas, etc.) (Francis et al., 2016; Lyons et al., 2016).

De la misma forma, en el día a día del centro se trata de asegurar que la comunicación es fluida, bidireccional y se comprende, algo que se cuida de forma especial en los casos de fami- lias inmigrantes, poniendo los medios oportunos para ello (por ejemplo, intérpretes) y atendiendo a que los documentos estén traducidos a los distintos idiomas de referencia de las familias (Labajos et al., 2012; Francis et al., 2016; Valenzuela y Sales, 2016). A la vez son conscientes del valor que tienen para ellas los intercambios informales con el profesorado (Egido y Bertran, 2017).

Todo ello contribuye a que las familias perciban el compromiso del equipo no solo con su hijo o hija, sino también con ellas. Se construyen relaciones fundamentadas en el respeto, apoyo y confianza mutua con el personal del centro, desde una posición de igualdad, en donde se valora lo que todos (profesionales y familias) pueden aportar (Francis et al., 2016; Lyons et al., 2016; Simón et al., 2016b; Thompson y Timmons, 2017).

Lo anterior coincide, en gran medida, con las reflexiones realizadas por Haines et al., (2015) quienes, en su análisis sobre la relación con las familias, identifican como características de centros que se consideran inclusivos, la existencia de una cultura escolar acogedora y atenta a la diversidad y un liderazgo fuerte con una clara comprensión del sentido y significado de la educación inclusiva que dirige sus acciones. Hacen referencia a una relación fundamentada en la confianza. Pero, además, la generación de oportunidades diversas y bidireccionales para la participación de las familias es un elemento importante, como ocurre en las experiencias revisadas.

\section{c) Las familias participan en la vida del centro}

Los centros escolares analizados parten de una visión amplia del concepto de colaboración adoptando, por tanto, un enfoque proactivo a este respecto (Egido y Bertran, 2017). Las familias participan en una diversidad de espacios como asambleas, tertulias dialógicas o en comisiones de trabajo para responder a necesidades del centro, el alumnado, las familias, etc. Están presentes en el aula en grupos interactivos u otras dinámicas (Fernández-Batanero, 2014; Flecha et al., 2011; García-Cano, Márquez y Domínguez, 2016; Iglesias et al., 2013; Simón et al., 2016b). También se cuenta con ellas en los procesos de transición entre etapas educativas (Martínez et al., 2012).

Además, se reconoce la necesidad de conformar comunidades de apoyo dentro y fuera de los centros y del horario escolar, creando una red de vínculos en los que se comparte información, preocupaciones, intereses o recursos desarrollando, para ello, diferentes acciones como, por ejemplo, actividades de aprendizaje servicio en beneficio del alumnado y del entorno (Escudero, 2009; Flecha et al., 2011). En este marco se encuentra la experiencia de los denominados Grupos de Ayuda Mutua (GAM) (Gallego, 2013; Gallego et al., 2018). Asimismo, se llevan a cabo actividades de formación de familiares, relacionadas tanto con cómo ayudar a sus hijos e hijas como con otros intereses de las familias (Labajos et al., 2012), o se promueven espacios de diálogo en torno a sus preocupaciones (Palou e Isern, 2008).

En estos centros se invita a que las familias asuman un liderazgo en la escuela (Francis et al., 2016). Esta implicación en la toma de decisiones relacionada con el proceso educativo, el desarrollo del currículo o la evaluación y la mejora de la escuela, se destaca como estratégica en estos centros (Francis et al., 2016; Martínez y Gómez, 2013). En definitiva, tienden a promover formas de participación con más posibilidades de contribuir al éxito escolar, de influir positivamente en el aprendizaje y la participación de los alumnos y alumnas, empoderando a las familias, y que en el proyecto INCLUD-ED (2011) denominan como decisoria, educativa y evaluativa. Pero, a la vez, son contextos educativos abiertos al entorno comunitario que buscan la colaboración de un conjunto amplio de entidades públicas y privadas para apoyar a estudiantes y familias (Egido y Bertran, 2017). Centros 
que se alinean en una estrategia coherente con los esfuerzos de otros actores locales como pueden ser empresas, grupos comunitarios, universidades o diferentes servicios públicos relacionados con las familias y la infancia (Ainscow et al., 2016).

Como se puede apreciar, la participación de las familias se considera un aspecto definitorio de estos centros. Por ello, es necesario calificar qué entendemos por participación en este marco.

\section{Cuando hablamos de participación de las familias en la es- cuela, ¿de qué estamos hablando?}

Como ya han venido señalando diferentes autores (UNICEF, 2014), bajo el mismo término de "participación" de las familias podemos encontrar diferentes formas de entenderlo y, en consecuencia, de concretarlo en la práctica. De forma que, incluso, en algunos casos un centro puede estar satisfecho con la participación de las familias cuando en realidad está haciendo referencia a actividades que, para otros, no se consideran como tal o se perciben como formas básicas o "simples" de participación.

Podemos entender la participación de las familias en la escuela como un proceso dirigido a un objetivo compartido (como es el de articular con equidad la presencia, el aprendizaje y la participación de todo el alumnado) y necesario para alcanzarlo con garantías, y que se fundamenta en la construcción de una relación de colaboración entre profesionales y familias. Para lograr esta finalidad hay que partir de cuatro premisas básicas. En primer lugar, a la escuela le corresponde promover una relación de colaboración y capacitar a las familias. Debe realizar esfuerzos específicos para promover su implicación (UNICEF, 2014), especialmente de aquellos grupos más vulnerables o marginados (IBE-UNESCO, 2016), y construir con ellas un modelo de corresponsabilidad educativa conforme proponen Collet-Sabet et al. (2014).

La segunda premisa tiene que ver la colaboración. El procedimiento para que la familia sea apoyo es construir una relación de colaboración en donde los agentes intercambian información y comparten conocimientos para trabajar juntos en la consecución de una meta compartida. En esta línea y conforme señalan
Turnbull et al. (2010), esta relación debe caracterizarse por la existencia de una comunicación fluida, amigable, clara, donde se comparta cuanta información se considere necesaria, en la que se perciba a los y las docentes como competentes y comprometidos con la defensa de los derechos del alumnado y sus familias, y fundamentada en el respeto y la confianza.

La tercera premisa se refiere al impacto en la calidad. En la medida que se introducen mejoras que facilitan la inclusión, se contribuye al bienestar y a la calidad de vida de todos los implicados (alumnado, familias y profesionales). Pero también mejora la actuación docente y a la calidad del centro en general.

La última premisa se refiere a la idea de proceso para enfatizar su función transformadora. Llama la atención sobre el papel que la familia debe tener hasta convertirse en agente activo para la transformación del centro, lo que supone una implicación real en las decisiones y en la vida escolar. La consideración de la participación de la familia como un proceso nos invita a pensarla como una secuencia que debe avanzar hacia mayores cuotas de implicación, en la línea del análisis de Susinos y Ceballos (2012) respecto a la participación e implicación de los estudiantes. En este caso, nos ha parecido inspiradora la escalera de participación ciudadana de Arnstein (1969). Esta autora propone tres grados de participación, comenzando por un nivel bajo de una especie de pseudoparticipación, donde los espacios son "ficticios"; un segundo nivel simbólico donde hay espacios de información, consulta e intercambio de propuestas, pero sin influencia en la toma de decisiones; y un tercer nivel que constituye la participación real. La Tabla 3 presenta nuestra propuesta adaptada a la participación de las familias en el marco de una escuela que se considera "inclusiva", donde el nivel superior implica su empoderamiento en diferentes grados. La colaboración escuela-familia se entiende que debe estar fundamentada en una relación que busca la promoción de una relación de igualdad, empezando por promover el equilibrio de poder entre los colaboradores, los cuales deben reconocerse mutuamente con igual capacidad y posibilidad, tanto para tomar la iniciativa, opinar y que su voz sea escuchada, como de influir y tomar parte en las decisiones.

Tabla 3.

Escala de participación de las familias.

COLABORACIÓN ESCUELAFAMILIAS

2 COALICIÓN DE LA ESCUELA CON LAS FAMILIAS

CONSULTA A LAS FAMILIAS

INFORMACIÓN A LAS FAMILIAS
Escuela y familia actúan indistintamente como corresponsables, promotores y en colaboración para derribar barreras y trabajar de manera sostenible y complementaria en la transformación del centro escolar, con la finalidad de ser más inclusivo. Familias empoderadas

La voz de las familias es considerada en la planificación de acciones, pero su participación está guiada por la escuela y mediada por los objetivos que esta se traza.

Se informa y consulta a las familias, pero estas tienen poca influencia en la toma de decisiones respecto a las acciones que lleva a cabo la escuela.

El papel de las familias se limita a receptores de información de las decisiones que adopta

la escuela. Se carece de espacios, canales o mecanismos para "proyectar su voz".

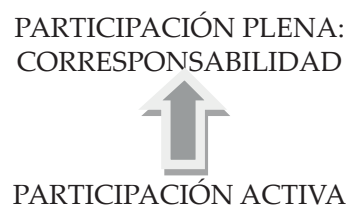

PARTICIPACIÓN PARCIAL

PARTICIPACIÓN SIMBÓLICA

PARTICIPACIÓN FICTICIA

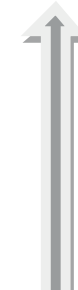

FORMA

DEFINICIÓN

NIVEL 


\section{Referencias bibliográficas}

Ainscow, M., Dyson, A., Goldrick, S., y Kerr, K. (2015). Learning from what works: Proposals for the reform of the English education system. Manchester: The Centre for Equity in Education at the University of Manchester.

Ainscow, M., Dyson, A., Hopwood, L., \& Thomson, S. (2016). Primary Schools Responding to Diversity: Barriers and Possibilities. York: Cambridge Primary Review Trust.

Alberta Education (2013). Indicators of Inclusive Schools: Continuing the Conversation. Alberta: Alberta Government. Recuperado de https://education.alberta.ca/media/482253/indicators_of_inclusive_schools.pdf

Arnstein, S. R. (1969). A Ladder of Citizen Participation. Journal of the American Planning Association, 35(4), 216-224. doi: http:// dx.doi.org/10.1080/01944366908977225

Booth, T., y Ainscow. M. (2015). Guía para la Educación Inclusiva. Desarrollando el aprendizaje y la participación en los centros escolares. Madrid: OEI/FUHEM [Trad. Cast. Booth, T. \& Ainscow, M. (2011). Index for Inclusion. Developing learning and participation in schools ( $3^{\mathrm{a}}$ ed.rev.).Bristol: CSIE]

Brown, R. I., Cobigo, V., \& Taylor, W. D. (2015). Quality of life and social inclusion across the lifespan: challenges and recommendations. International Journal of Developmental Disabilities, 61(2), 93-100. doi: http://dx.doi.org/10.1179/204738691 4Z.00000000092

Collet-Sabé, J., Besalú, X., Feu, J., y Tort, A. (2014). Escuelas, familias y resultados académicos. Un nuevo modelo de análisis de las relaciones entre docentes y progenitores para el éxito de todo el alumnado. Profesorado. Revista de Currículum y Formación de Profesorado, 18(2), 7-33.

CSIE (2016). Equality: Making It Happen. A guide to help schools ensure everyone is safe, included and leaning. Bristol: CSIE.

De Verdier, K., \& Ek, U. (2014). A Longitudinal Study of Reading Development, Academic Achievement, and Support in Swedish Inclusive Education for Students with Blindness or Severe Visual Impairment. Journal of Visual Impairment $\mathcal{E}$ Blindness, 108(6), 461-472.

Echeita, G. (2017). Educación inclusiva. Sonrisas y Lágrimas. Aula Abierta, 46, 17-24. doi: https://doi.org/10.17811/rifie.46.2017.17-24

Egido, I., y Bertran, M. (2017). Prácticas de colaboración familia-escuela en centros de éxito de entornos desfavorecidos. Pedagogía Social. Revista Interuniversitaria, 29, 97-110. doi: http://doi.org/ 10. SE7179/PSRI_2017.29.07

Epstein, J. (2011). School, family, and community partnerships: Preparing educators and improving schools (2nd.ed.). Boulder, CO: Westview Press.

Escudero, J. M. (2009). Buenas prácticas y programas extraordinarios de atención al alumnado en riesgo de exclusión educativa. Revista de Currículum y Formación del Profesorado, 13(3), 108-141.

Esteban-Guitart, M., Oller, J., y Vila, I. (2012). Vinculando escuela, familia y comunidad a través de los fondos de conocimiento e identidad. Un estudio de caso con una familia de origen marroquí. Revista de Investigación en Educación, 10(2), 21-34.

Fernández-Batanero, J. M. (2014). Strategies for inclusion in the face of social exclusion. Case study in Andalusia (Spain). European Journal of Special Needs Education, 29(3), 415-428. doi: https://doi.org/10.1080/08856257.2014.906978

Flecha, A., García, R., \& Rudd, R. (2011). Using Health Literacy in School to Overcome Inequalities. European Journal of Education, 46(2), 209-218. doi: http://dx.doi.org/10.1111/j.14653435.2011.01476.x
Francis, G. L., Gross, J. M. S., Blue-Banning, M., Haines, S., \& Turnbull A. P. (2016). School Principals and Parents Who Achieve Optimum Results: Lessons Learned from 6 North American Schools that have Implemented Inclusive Practices. Revista Latinoamericana de Educación Inclusiva, 10(1), 43-60.

Gallego, C. (2013). Una formación compartida entre familias y profesores para el desarrollo de grupos de apoyo mutuo. Revista de Investigación en Educación, 11(3), 109-119.

Gallego, C., Jiménez, A., y Corujo, C. (2018). Otra forma de desarrollar el apoyo inclusivo: los Grupos de Apoyo Mutuo. Revista de Investigación en Educación, 16(2), 106-120.

García-Cano, M., Márquez, E., y Antolínez, I. (2016). Una comunidad de aprendizaje desde el enfoque intercultural: Diálogos, emergencias y contradicciones en la práctica escolar. Educación XX1, 19(2), 251-271. doi: http://dx.doi.org/10.5944/ educXX1.13940

Haines, S. J., Gross, J. M. S., Blue-Banning, M., Francis, G., y Turnbull, A. (2015). Fostering Family-School and Community-School Partnership in Inclusive Schools: Using practice as a Guide. Research and Practice for People with Severe Disabilities, 40(3), 227-239. doi: http://dx.doi. org/10.1177/1540796915594141

Harris, J., Carrington, S., \& Ainscow, M. (2017). Promoting Equity in Schools. Collaboration, Inquiry and Ethical Leadership. Abingdon: Routledge

Hehir, T., Grindal, T., Freeman, B., Lamoreau, R., Borquaye, Y., \& Burke, S. (2016). A summary of the evidence on inclusive education. São Paulo: Alana-Abt.

IBE-UNESCO (2016). Training Tools for Curriculum Development. Reaching out to all Learners: a Resource Pack Supporting Inclusive Education. Geneva: International Bureau of Education-UNESCO. Recuperado de http://www.ibe.unesco.org/sites/default/ files/resources/ibe-crp-inclusiveeducation-2016_eng.pdf

Iglesias, B., de la Madrid, L., Ramos, A., Robles, C., y Serrano, A. (2013). Metodologías innovadoras e inclusivas en educación secundaria: los grupos interactivos y la asamblea de aula. Tendencias Pedagógicas, 22, 111-126.

INCLUD-ED (2011). Actuaciones de éxito en las escuelas europeas. Madrid: Secretaría General Técnica. Ministerio de Educación.

Labajos, S., Gallego, B., y Lago, M. (2012). Factores de la cultura escolar que favorecen o dificultan la inclusión del alumnado de diversidad cultural. propuestas para el plan de acogida. TABANQUE. Revista Pedagógica, 25, 127-150

Leiva, J. J., y Escarbajal A. (2011). La participación de las familias inmigrantes como fundamento pedagógico en la construcción de la interculturalidad en la escuela. Educatio Siglo XXI, 29(2), 389-416.

Lyons, W., Thompson, S. A., \& Timmons, V. (2016). “We are inclusive. We are a team. Let's just do it." Commitment, collective efficacy, and agency in four inclusive schools. International Journal of Inclusive Education, 13, 889-907. doi: https://doi. org/10.1080/13603116.2015.1122841

Martínez, D. C., Conroy, J. W., \& Cerreto, M .C. (2012). Parent Involvement in the Transition Process of Children With Intellectual Disabilities: The Influence of Inclusion on Parent Desires and Expectations for Postsecondary Education. Journal of Policy and Practice in Intellectual Disabilities, 9(4), 279-288.

Martínez, A., y Gómez, J.L. (Coord.) (2013). Escuelas inclusivas singulares. Madrid: Grupo5.

Martínez-González, R. A., Rodríguez-Ruiz, B., y Gimeno, J. L. (2010). Áreas de cooperación entre los centros docentes y las familias. Estudio de caso. Educatio Siglo XXI, 28(1),127-156.

Martínez-Figueira, M. E., Páramo-Iglesias, M. B., y de Matos Claudino, E. M. (2015). Desafíos actuales a la inclusión: un 
estudio de caso en un aula de preescolar portuguesa. Revista Actualidades Investigativas en Educación, 15(1), 1-18. doi: http:// doi.org/10.15517/aie.v15i1.17736

Palou, S. e Isern A. (2008). La complicidad de las familias. Cuadernos de Pedagogía, 378, 61-63.

Parrilla, M. A., Muñoz M. A., y Sierra, S. (2013) Proyectos educativos con vocación comunitaria. Revista de Investigación en Educación, 11(3), 15-31.

Parsons, A., Walsemann, K. M., Jones, S. J., Knopf, H., \& Blake, C. (2018). Parental Involvement: Rhetoric of Inclusion in an Environment of Exclusion. Journal of Contemporary Ethnography, 47(1), 113-139. doi: https://doi.org/ 10.1177/0891241616676874

Porter, G., \& Smith, D. (2011). Exploring Inclusive Educational Practices Through Professional Inquiry. Rotterdman: Sebse Publishers.

Porter, G., y Towell, D. (2017). Promoviendo la educación inclusiva. Claves para el cambio transformacional en los sistemas de educación. Canada: Centre for Inclusive Futures.

Puigdellívol, I., Molina, S., Sabando, D., Gómez-Zepeda, G., \& Petreñas, C. (2017). When community becomes an agent of educational support: communicative research on Learning Communities in Catalonia. Disability \& Society, 32(7), 10651084. doi: http://dx.doi.org/10.1080/09687599.2017.1331835

Rodrigo, Ma. J., Martínez-González, R. A., y Rodríguez-Ruiz, B. (2018) La relación centro escolar-familia como factor protector de conductas transgresoras en la adolescencia. Aula Abierta, 47(2), 149-158. doi: https://doi.org/10.17811/rifie.47.2.2018.149-158

Simón, C., Giné, C., y Echeita, G (2016a). Escuela, familia y comunidad: Construyendo alianzas para promover la inclusión. Revista Latinoamericana de Inclusión Educativa, 10(1), 25-42.

Simón, C., Sandoval, M., Echeita, G., Calero, C., Núñez, B., Sotto de, P., Pérez ,M. M., y García de la Torre, A.B (2016b). Transformando la "gramática escolar" para ser más inclusivos: La experiencia de tres centros educativos. Contextos Educativos. Revista de Educación, 19, 7-24.

Susinos, T., y Ceballos, N. (2012). Voz del alumnado y presencia participativa en la vida escolar. Apuntes para una cartografía de la voz del alumnado en la mejora educativa. Revista de Educación, 359, 24-44. doi: http://dx.doi.org/10.4438/1988592X-RE-2012-359-194

Thompson, S. A., \& Timmons, V. (2017). Authentic Inclusion in Two Secondary Schools: "It's the Full Meal Deal. It's Not Just in the Class. It's Everywhere". Exceptionality Education International, 27(1), 62-84.

Turnbull, A. P., Turnbull, H. R., Erwin, E., Soodak, L., \& Shogren, K.E. (2010). Families, professionals, and exceptionality. Positive outcomes through partnership and trust (6a Ed.). Upper Saddle River, NJ: Pearson/ Merrill-Prentice Hall.

UNESCO (2005) Guidelines for inclusion: Ensuring Access to Education for All. París: UNESCO. Recuperado de http://unesdoc. unesco.org/images/0014/001402/140224e.pdf

UNESCO (2016). Educación para transformar vidas. Metas, opciones de estrategia e indicadores. OREALC/UNESCO Santiago. Recuperado de http://unesdoc.unesco.org/images/0024/002452/245278s.pdf

UNICEF (2014). Parents, Family and Community Participation in Inclusive Education: Webinar 13 Companion Technical Booklet. Nueva York: UNICEF.

Valenzuela, C., y Sales, A. (2016). Los efectos de la participación familiar dentro del aula ordinaria. Revista Nacional e Internacional de Educación Inclusiva, 9(2), 71-86.

Vigo, B., Dieste, B., y Thurtson, A. (2016). Aportaciones de un estudio etnográfico sobre la participación de las familias a la formación crítica del profesorado en una escuela inclusiva. Revista Electrónica Interuniversitaria de Formación del Profesorado, 19(1), 1-14. doi: http://dx.doi.org/10.6018/reifop.19.1.246341 